\title{
Tinea Corporis with Trichophyton Rubrum Mimicking a Flare-Up of Psoriasis UnderTreatment with IL17-Inhibitor Ixekizumab
}

\author{
Vladimir Emelianov Laurence Feldmeyer Nikhil Yawalkar \\ Kristine Heidemeyer \\ Department of Dermatology, Inselspital, Bern University Hospital, University of Bern, Bern, \\ Switzerland
}

\section{Keywords}

Psoriasis $\cdot$ IL-17-inhibitor $\cdot$ Ixekizumab $\cdot$ Tinea

\begin{abstract}
Biologics, as IL17 inhibitors, are frequently prescribed for moderate to severe plaque psoriasis. Although mucocutaneous candida infections are a well-known side effect of IL-17 inhibitors, there is no data about dermatophyte infection under this therapy. Generalized tinea corporis can mimic the clinical picture of psoriasis, especially if concomitant treatment with topical corticosteroids is used. Therefore, physician should be aware of this differential diagnosis if they suspect a loss of efficacy of IL-17-inhibitors with a flare-up of psoriasis.
\end{abstract}

(C) 2021 The Author(s).

Published by S. Karger AG, Basel

\section{Introduction}

Ixekizumab, a high-affinity humanized monoclonal antibody targeting IL-17A, is approved for the treatment of plaque psoriasis and psoriasis arthritis and has shown superiority in short time efficacy over ustekinumab and even guselkumab in psoriasis $[1,2]$. Ixekizumab has good tolerability, with the main side effects being injection-site reactions, nasopharyngitis, and candida infections [3]. Here, we report a case of tinea corporis with Trichophyton rubrum imitating psoriasis flare-up undertreatment with ixekizumab. 

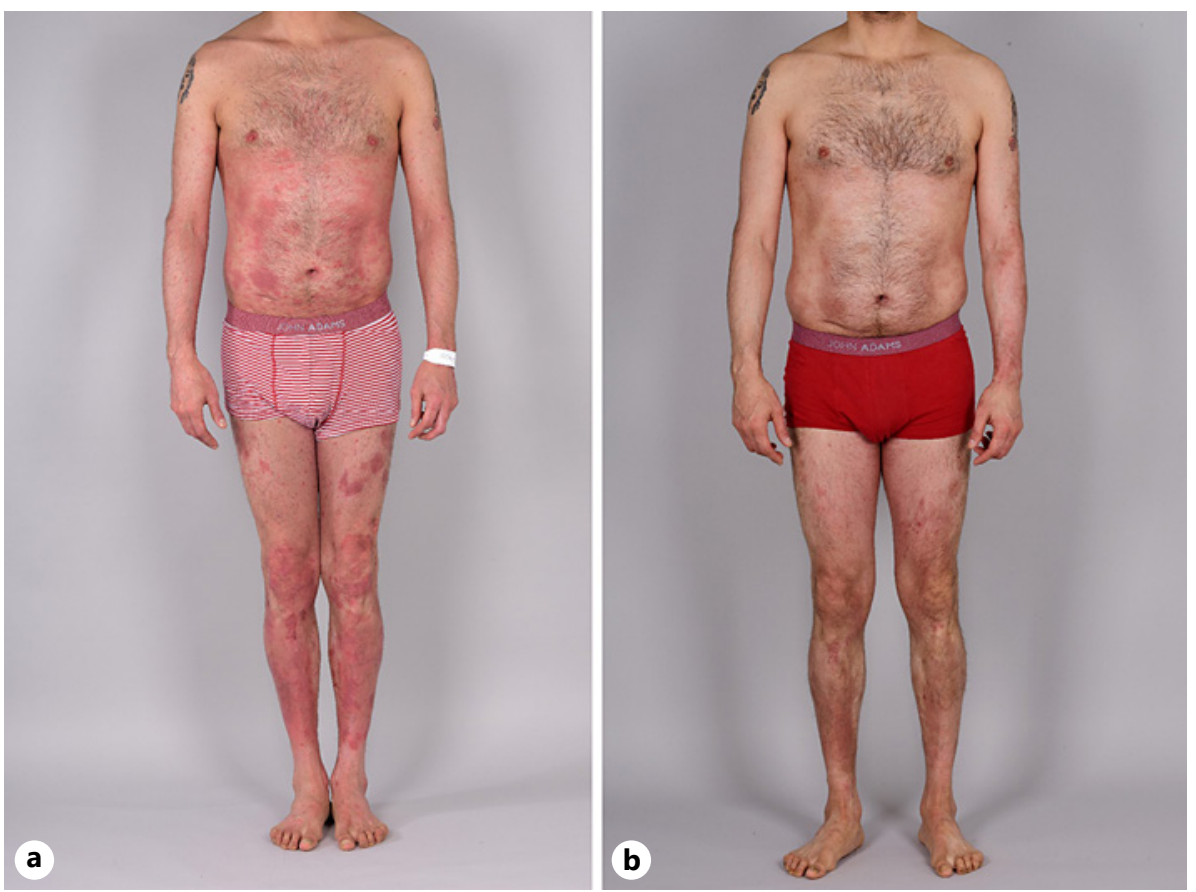

Fig. 1. The patient with severe plaque psoriasis before treatment (a); the patient after 3 months of ixekizumab (b).

\section{Case Report}

A 44-year-old male patient with a history of plaque psoriasis for over 20 years and lack of treatment response to standard topical treatments, phototherapy (UVB), and apremilast was initiated on therapy with ixekizumab. After 3 months of treatment, he showed an excellent response with a decrease in PASI score from 22.6 to 1.5 and daily life quality index from 18 to 2 points (Fig. 1a, b).

After 10 months of treatment, the patient presented to our outpatient clinic with a presumed relapse of psoriasis with widely spread itchy erythrosquamous papules, plaques, and onychodystrophy affecting all of his finger and toenails. Therapy with topical calcipotriol and corticosteroids led to further spread of the lesions with a centrifugal pattern with isolated pustules (Fig. 2a). Histopathologic examination showed a perifollicular lymphohistiocytic infiltrate with epithelioid and giant cells, as well as fungal spores. T. rubrum was detected in the culture of skin scrapings.

Since the dermatophyte infection was wide-spread and his psoriasis well-controlled, ixekizumab was discontinued, and antifungal treatment with terbinafine ( $250 \mathrm{mg} /$ day), topical ciclopirox (1\%) cream, and ketoconazole (2\%) shampoo was initiated. After 6 weeks, a complete remission of skin lesions and itch was observed. The microbiologic analysis of skin scrapings was negative. Therapy with ixekizumab was restarted and has been well-tolerated with excellent effectiveness (absolute PASI <1) for over 8-month follow-up (Fig. 2b).

\section{Discussion}

Psoriasis is a chronic immune-mediated inflammatory skin disease with a genetic predisposition and several comorbidities, including arthritis, metabolic syndrome, and autoimmune diseases [4]. About $20 \%$ of these patients have a moderate to severe manifestation, demanding a systemic treatment, including biologics like ixekizumab [5]. 

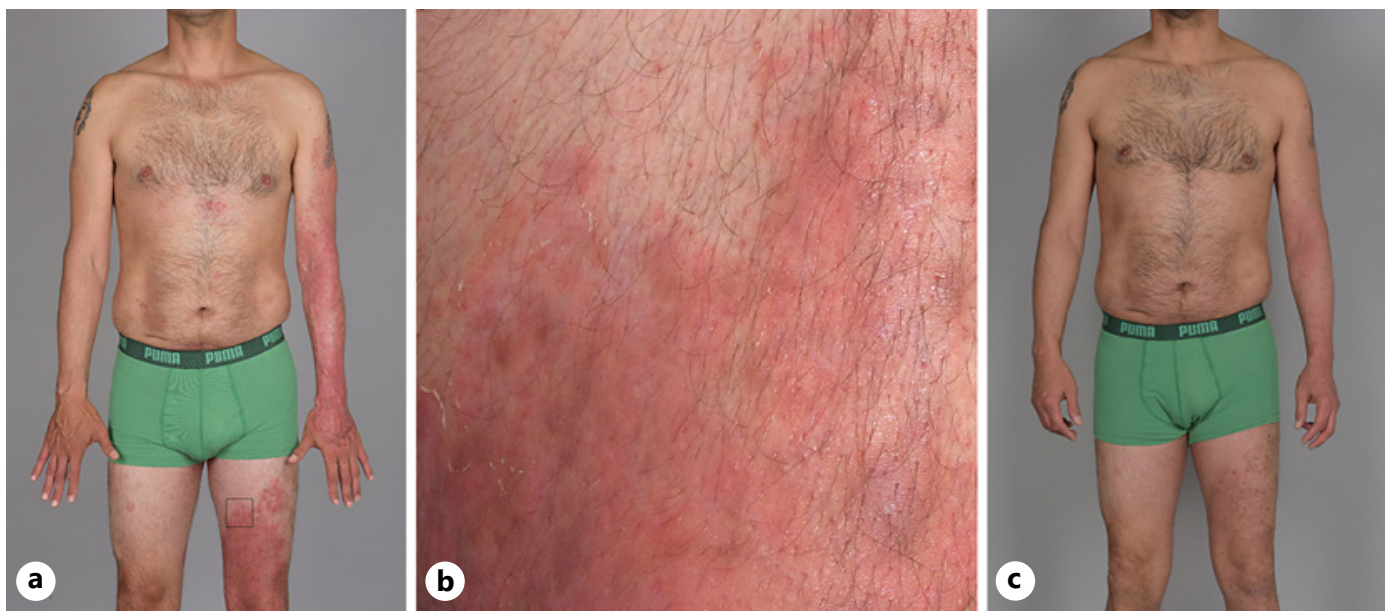

Fig. 2. Flare-up of erythrosquamous plaques (a); close-up of marked lesion (b), after treatment with topical and systemic antifungal therapy (c).

Several studies have described high and rapid efficacy for ixekizumab, with $88.7 \%$ reaching a PASI 75 and 69.9\% reaching a PASI 90 already at week 12 [5]. Ixekizumab has a favorable safety profile, with main side effects of nasopharyngitis, upper respiratory tract infections, injections-site reactions, and arthralgias [6]. In a survey with over 17,000 patientyears of exposure, Armstrong et al. [7] described the following infections under the treatment with ixekizumab: nasopharyngitis (8.9/100 p-y), upper respiratory tract infection (5.4/100 p-y), bronchitis (2.3/100 p-y), sinusitis (2.2/100 p-y), urinary tract infection (2.0/100 p-y), candidiasis (1.9/100 p-y), influenza (1.8/100 p-y), pharyngitis (1.6/100 p-y), gastroenteritis 1.4/100 p-y), cellulitis (0.2/100 p-y), pneumonia 0.1/100 p-y), appendicitis (0.1/100 p-y), and erysipelas $0.1 / 100 \mathrm{p}-\mathrm{y})$. Compared to other biologics, a higher number of mucocutaneous Candida infections has been observed in patients treated with ixekizumab (3.8-5.5\%) [6,7]. The frequency of candidiasis was highest in the first year (3.2/100 patient-years) and then ranged from 2.1 to 2.3 per $100 \mathrm{p}-\mathrm{y}$ in subsequent years [7]. This phenomenon is explainable by the mode of action of ixekizumab and the well-known importance of IL-17 in fungal immunity [7].

TH-17 cells, producing IL-17A, IL-17F, and IL-22, are described to play a crucial role in antifungal immunity in barrier tissues [8]. Patients with defects affecting the TH17 immunity pathway are unable to clear superficial candida and dermatophyte infections, which has been well demonstrated in patients with chronic mucocutaneous candidiasis due to autosomal recessive IL-17RA deficiency and autosomal dominant IL-17F deficiency $[8,9]$.

Although the risk of candidiasis undertreatment with IL-17 blockers is well described, scanty data is available regarding dermatophyte infections. Undertreatment with secukinumab 2 cases of perianal dermatophyte infections have been published, and 4 cases of tinea pedis were observed in the Efficacy of Response and Safety of 2 Fixed Secukinumab Regimens in Psoriasis study [10]. Furthermore, one case report of tinea incognito undertreatment with brodalumab was described in literature [11]. In addition, Strober et al. [12] reported that tinea pedis under ixekizumab therapy occurred in 1.3/100 p-y (7 ixekizumab trials, total 6479.8 py). However, no data on disseminated dermatophyte infections with ixekizumab have been published so far.

Loss of efficacy of ixekizumab is rare but occurred in $~ 2 \%$ of patients in a 108-week follow-up study [6]. Nevertheless, if patients treated with IL-17 inhibitors show a new rash of erythrosquamous itchy plaques, a generalized dermatophyte infection should be considered, 
and microbiological examination should be performed. The use of topical steroids by the patients can make the clinical diagnosis especially difficult. In conclusion, our observation reminds us that clinicians must maintain a high index of suspicion for fungal infections, including dermatophytes, in patients receiving IL-17 antagonists.

\section{Statement of Ethics}

The patient has given written informed consent to publish his case including publication of images. Research complies with the guidelines for human studies and was conducted ethically in accordance with the World Medical Association Declaration of Helsinki.

\section{Conflict of Interest Statement}

Nikhil Yawalkar and Kristine Heidemeyer have served as consultants for Eli Lilly. The remaining authors declare no conflict of interest.

\section{Funding Sources}

No funding for this case report.

\section{Author Contributions}

The first author (Vladimir Emelianov) and the coauthors (Laurence Feldmeyer, Nikhil Yawalkar, Kristine Heidemeyer) were all involved in summarizing this case report, writing the manuscript and have proof read the final version of the manuscript.

\section{References}

1 Reich K, Pinter A, Lacour JP, Ferrandiz C, Micali G, French LE, et al. Comparison of ixekizumab with ustekinumab in moderate-to-severe psoriasis: 24-week results from IXORA-S, a phase III study. Br J Dermatol. 2017 Oct; 177: 1014.

2 Blauvelt A, Papp K, Gottlieb A, Jarell A, Reich K, Maari C, et al. A head-to-head comparison of ixekizumab vs. guselkumab in patients with moderate-to-severe plaque psoriasis: 12-week efficacy, safety and speed of response from a randomized, double-blinded trial. Br J Dermatol. 2020;182(6):1348-58.

3 Farahnik B, Beroukhim K, Zhu TH, Abrouk M, Nakamura M, Singh R, et al. Ixekizumab for the treatment of psoriasis: a review of phase III trials. Dermatol Ther. 2016;6(1):25-37.

4 Rendon A, Schäkel K. Psoriasis pathogenesis and treatment. Int J Mol Sci. 2019;20(6):1475.

5 Papp KA, Leonardi CL, Blauvelt A, Reich K, Korman NJ, Ohtsuki M, et al. Ixekizumab treatment for psoriasis: integrated efficacy analysis of three double-blinded, controlled studies (UNCOVER-1, UNCOVER-2, UNCOVER-3). Br J Dermatol. 2018;178(3):674-81.

6 B Blauvelt A, Gooderham M, Iversen L, Ball S, Zhang L, Agada NO, et al. Efficacy and safety of ixekizumab for the treatment of moderate-to-severe plaque psoriasis: results through 108 weeks of a randomized, controlled phase 3 clinical trial (UNCOVER-3). J Am Acad Dermatol. 2017;77(5):855-62.

7 Armstrong A, Paul C, Puig L, Boehncke WH, Freeman M, Torii H, et al. Safety of ixekizumab treatment for up to 5 years in adult patients with moderate-to-severe psoriasis: results from greater than 17,000 patient-years of exposure. Dermatol Ther. 2020;10(1):133-50.

8 Lilic D. Unravelling fungal immunity through primary immune deficiencies. Curr Opin Microbiol. 2012;15(4): 420-6.

9 Cypowyj S, Picard C, Maródi L, Casanova JL, Puel A. Immunity to infection in IL-17-deficient mice and humans. Eur J Immunol. 2012;42(9):2246-54. 
10 Quach OL, Hsu S. Perianal dermatophytosis during secukinumab therapy for plaque psoriasis. JAMA Dermatol. 2016 Apr;152(4):486-7.

11 DiRuggiero D. Successful management of psoriasis and treatment-induced tinea incognito: a case report. J Clin Aesthet Dermatol. 2020;13(9 Suppl):S21-5.

12 Strober B, Leonardi C, Papp KA, Mrowietz U, Ohtsuki M, Bissonnette R, et al. Short- and long-term safety outcomes with ixekizumab from 7 clinical trials in psoriasis: etanercept comparisons and integrated data. J Am Acad Dermatol. 2017 Mar;76(3):432-440. 